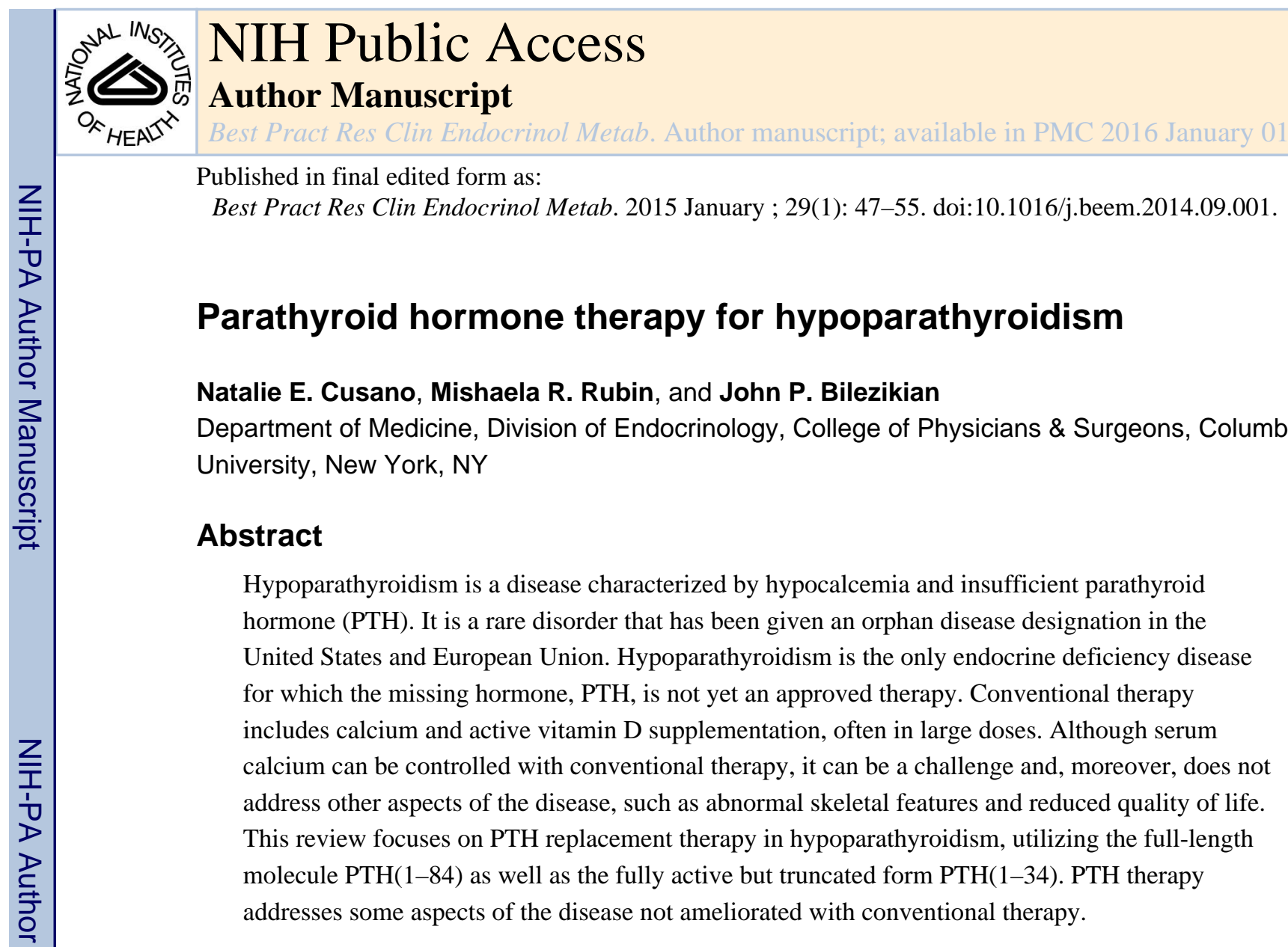

Key Terms

Hypoparathyroidism; Parathyroid Hormone; PTH(1-34); Teriparatide; PTH(1-84)

\title{
Introduction
}

Hypoparathyroidism is a disease characterized by hypocalcemia and insufficient parathyroid hormone (PTH). Many patients also demonstrate hyperphosphatemia and hypercalciuria. The acute clinical manifestations relate to symptoms of hypocalcemia and neuromuscular irritability, including muscle cramps and paresthesias. Life-threatening heart arrhythmias, laryngospasm and seizures can occur. Long-term complications include nephrocalcinosis, nephrolithiasis or renal failure; soft-tissue calcifications in the basal ganglia, other brain compartments, or the vasculature itself; neurocognitive complaints and reduced quality of

() 2014 Elsevier Ltd. All rights reserved.

Corresponding author: Natalie E. Cusano, M.D., Columbia University College of Physicians \& Surgeons, Division of Medicine, 630 West $168^{\text {th }}$ Street, PH 8W-864, New York, NY 10032, nc2433@ cumc.columbia.edu, Fax: (212) 305-6486, Phone: (212) 305-2801. Mishaela R. Rubin, M.D., Columbia University College of Physicians \& Surgeons, Division of Medicine, 630 West $168^{\text {th }}$ Street, PH 8W-864, New York, NY 10032, mrr6@ cumc.columbia.edu, Fax: (212) 305-6486, Phone: (212) 305-7859

John P. Bilezikian, M.D., Columbia University College of Physicians \& Surgeons, Division of Medicine, 630 West $168^{\text {th }}$ Street, PH 8W-864, New York, NY 10032, jpb2@cumc.columbia.edu, Fax: (212) 305-6486, Phone: (212) 305-6257

Publisher's Disclaimer: This is a PDF file of an unedited manuscript that has been accepted for publication. As a service to our customers we are providing this early version of the manuscript. The manuscript will undergo copyediting, typesetting, and review of the resulting proof before it is published in its final citable form. Please note that during the production process errors may be discovered which could affect the content, and all legal disclaimers that apply to the journal pertain.

Disclosures

No disclosures are reported for Dr. Cusano. 
life; and abnormally low bone turnover [1-4]. While patients with hypoparathyroidism often have bone mineral density values higher than healthy controls, there is some evidence that vertebral fracture risk may be increased [5], although overall fracture risk may be similar to age-matched controls [6].

Hypoparathyroidism is rare, with an estimated 59,000 individuals in the United States suffering from the disorder [7]. It has been given an orphan disease designation by the United States Food and Drug Administration and the European Commission. The most common cause is inadvertent removal or irreversible damage to the parathyroid glands during thyroid or other neck surgery [1]. Other causes include autoimmune disease and rare genetic disorders such as DiGeorge syndrome, familial isolated hypoparathyroidism, autoimmune polyglandular syndrome type 1 and autosomal dominant hypocalcemia [8,9]. Severe magnesium deficiency is the only reversible cause of hypocalcemia with low PTH concentrations through impairment of PTH release and PTH resistance [1].

Hypoparathyroidism is the only endocrine deficiency disease for which the missing hormone, PTH, is not yet an approved therapy. This review focuses on the use of PTH treatment in hypoparathyroidism, in the form of the full-length molecule PTH(1-84) as well as the fully active but truncated form $\mathrm{PTH}(1-34)$.

\section{Treatment of hypoparathyroidism}

There are no formal guidelines to assist in management decisions for patients with hypoparathyroidism. Intravenous calcium may be necessary in the acute setting. Conventional therapy in the outpatient setting includes calcium and active vitamin D supplementation, often in large doses. Maintaining serum calcium within an acceptable range must be balanced against the development of hypercalciuria and the presence of hyperphosphatemia. Serum calcium often fluctuates in hypoparathyroid patients on conventional therapy, requiring adjustments in the supplementation regimen. Thiazide diuretics may be a useful adjunct in the setting of significant hypercalciuria [1].

While Fuller-Albright first considered the use of a parathyroid extract in hypoparathyroid subjects in 1929 [10], this research was abandoned for many years until the past several decades when PTH became available as a potential therapeutic agent. The theoretical advantages of PTH over conventional therapy in the management of hypoparathyroidism include: a reduction in the amounts of calcium and vitamin $\mathrm{D}$ requirements, reduction in urinary calcium, improvement in quality of life, reduction in ectopic soft tissue calcification, and improvement in abnormal bone remodeling dynamics. PTH has been investigated as a therapy for hypoparathyroidism in the form of the full-length molecule PTH(1-84) [11-14] as well as the fully active but truncated form $\mathrm{PTH}(1-34)$ [15-18]. Both formulations are administered as a subcutaneous injection. In the studies investigating PTH(1-34), the dose of PTH was titrated to achieve independence from active vitamin D therapy. The pharmacokinetics of PTH(1-34) are relatively short, requiring multiple injections per day. In the studies investigating PTH(1-84), PTH was used as an add-on to standard therapy. The pharmacokinetics of $\mathrm{PTH}(1-84)$ are relatively long, with once daily or every other day 
injections possible. The long-term studies investigating $\mathrm{PTH}(1-34)$ and $\mathrm{PTH}(1-84)$ therapy of hypoparathyroidism are summarized in Table 1.

\section{Effects of PTH therapy on supplementation requirements and serum calcium}

In the studies of PTH(1-34), the drug was titrated to maintain serum calcium in the intended low-normal range off active vitamin D therapy. The studies investigating $\mathrm{PTH}(1-84)$ have used either fixed doses or doses titrated to maintain biochemistries. All studies have demonstrated that serum calcium was maintained in the setting of a reduction or elimination of calcium and active vitamin D supplementation [11-18].

The pivotal trial of PTH(1-84) therapy is the REPLACE trial [14], a multicenter study of 134 subjects randomized to $\mathrm{PTH}(1-84)(\mathrm{n}=90)$ or placebo $(\mathrm{n}=44)$. Subjects were initially treated with PTH(1-84) at a dose of $50 \mu \mathrm{g}$ daily, titrated as needed to $75 \mathrm{or} 100 \mu \mathrm{g}$. The primary outcome was a composite of the proportion of patients at week 24 who achieved at least a 50\% reduction in both oral calcium and active vitamin D supplementation while maintaining a serum calcium of at least the subject's baseline concentrations and less than or equal to the upper limit of normal. 48 (53\%) of subjects in the PTH arm achieved this primary outcome compared to $1(2 \%)$ in the placebo arm $(\mathrm{p}<0.0001)$. The mean decrease in calcium supplementation in the PTH arm was 52\% and the mean decrease in active vitamin D supplementation was $78 \%$. Serum calcium was maintained despite the decrease in calcium and active vitamin D supplementation.

Data from the studies of Winer and colleagues through 3 years of PTH(1-34) therapy [15, 16] and the Columbia cohort through 4 years of PTH(1-84) therapy [12] demonstrates that serum calcium concentrations are maintained long-term in the setting of decreased calcium and vitamin $\mathrm{D}$ requirements.

\section{Effects of PTH therapy on urinary calcium}

PTH is necessary for the conservation of calcium in the proximal and distal renal tubules and many patients with hypoparathyroidism have hypercalciuria which can lead to nephrocalcinosis, nephrolithiasis, and renal failure [1]. Data regarding the efficacy of PTH therapy to decrease urinary calcium have been mixed (Table 1). The only study of PTH(134) therapy to show a decrease in urinary calcium was the open-label crossover trial of Winer and colleagues investigating PTH(1-34) administered continuously by pump versus PTH(1-34) subcutaneously twice daily [17]. In this study, urinary calcium was decreased significantly by $59 \%$ during the pump arm. The dramatic decrease in urinary calcium while patients were administered continuous PTH may indicate that more physiologic delivery of PTH to the renal tubules improves calcium reabsorption from the kidney on a more consistent basis. In the Columbia cohort, urinary calcium was significantly decreased during years 1 to 3 of the study, and while urinary calcium was below pre-treatment values at year 4 this difference did not meet statistical significance [12]. In the REPLACE trial, urinary calcium was not different between the PTH and placebo groups at study conclusion [14]. 


\section{Effects of PTH therapy on quality of life}

Hypoparathyroid patients often describe symptoms of "brain fog" and other neurocognitive complaints [1]. Using well-defined metrics, hypoparathyroid patients on conventional therapy have compromised quality of life compared to normal subjects despite eucalcemia [19-22]. There has been no formal evaluation of PTH(1-34) therapy on quality of life. Two groups have investigated the effect of $\mathrm{PTH}(1-84)$ therapy on quality of life using the same metric [20-22].

In the Columbia cohort, hypoparathyroid subjects received open-label PTH(1-84) through 5 years [20,22]. At baseline, subjects scored significantly lower than the normative reference range. With PTH therapy, intention-to-treat analysis showed significant improvement in mental and physical health that occurred early and persisted through 5 years. These data have a number of limitations, the most important one that it was open-label without a control group. The fact that these findings were sustained over 5 years, however, makes them less likely to be solely due to a placebo effect, although it does not remove the possibility of confounding. An important point is that in this cohort the dose of PTH was titrated to biochemical parameters. While serum calcium was maintained in the intended low-normal range for a majority of the subjects, the proportion of subjects with serum calcium levels within the normal reference range was much lower. Hypercalcemia was relatively rare during this study.

The only other study to address the effect of PTH(1-84) therapy on quality of life is that of Sikjaer and colleagues [21]. The investigation was randomized, placebo-controlled trial over 24 weeks with a fixed dose of PTH(1-84). They found that quality of life was improved in both the PTH and placebo arms without between-group differences. They had a relatively high incidence of hypercalcemia during their study, however, and the authors posited that the large fluctuations in serum calcium may have negated a possible benefit of PTH therapy. Considering that hypoparathyroid subjects may acclimate to relatively lower serum calcium values, these results may indicate that the reference range for serum calcium in normal subjects may not be suitable for all hypoparathyroid patients with regard to their mental and physical wellbeing.

Further studies are needed in a controlled manner to determine more definitely whether PTH is associated with improved quality of life.

\section{Effects of PTH therapy on extraskeletal calcifications}

Studies have demonstrated a reduction in serum phosphorus levels with $\mathrm{PTH}(1-84)$ therapy [11-14], although a decline in the calcium-phosphorus product has not been consistently seen. Any potential effect of PTH therapy on extra-skeletal calcifications remains unresolved. 


\section{Effects of PTH therapy on skeletal parameters}

\section{Bone density}

Bone density in hypoparathyroid patients is often above that for normal controls [1]. The data regarding the effect of PTH therapy on bone density is inconsistent. Studies using PTH(1-34) have not demonstrated a difference in bone density in PTH-treated subjects compared to subjects treated with conventional therapy [15-18]. The study of Sikjaer and colleagues showed small but significant decreases at the lumbar spine (-1.8\%) and hip $(-1.6 \%)$ without a difference at the forearm at 24 weeks [13]. In the Columbia cohort, bone density was significantly increased at the lumbar spine by $5.5 \%$ at 4 years; bone density at the hip sites was unchanged and at the forearm there was a significance decrease of $2.0 \%$ at 2 years without further decline [12].

\section{Dynamic parameters}

Bone turnover markers in hypoparathyroid subjects on conventional therapy are suppressed or in the low-end of the normal range [15, 16, 18, 23]. In all studies of PTH(1-34) and PTH(1-84) measuring serum and urine markers of bone turnover, these markers increase early during the treatment course $[12,15,16,18,23]$. In the Columbia cohort, markers of bone formation and resorption increased after initiation of $\mathrm{PTH}(1-84)$ therapy, with peak values up to 3-fold baseline at approximately 12 months [12]. After this period of heightened responsiveness, bone turnover markers decreased and at about 30 months reached a new steady state that was above baseline and more consistent with euparathyroid levels.

Tetracycline-labeled bone biopsies of hypoparathyroid subjects on conventional therapy demonstrate very few tetracycline labels consistent with a low bone turnover state [18, 24]. With both PTH(1-34) and PTH(1-84) therapy, histomorphometric analysis of bone biopsy specimens demonstrates a dramatic increase in the number of single and double tetracycline labels, as well as an increase in bone formation rate and mineralizing surface [18, 24].

\section{Structural parameters}

In hypoparathyroid subjects on conventional therapy, analysis of bone biopsy specimens shows that there is too much bone mineral in both the cortical and trabecular compartments $[18,23,25]$. With PTH(1-34) and PTH(1-84) therapy, there is an increase in the number of trabeculae and a decrease in trabecular thickness associated with intrabecular tunneling [18, 23, 25]. Intratrabecular tunneling is a phenomenon by which a thick trabecula splits into two or more thinner trabeculae and can also be seen in patients receiving PTH therapy for osteoporosis [26, 27]. In addition, there is increased osteoid and a decrease in trabecular spacing. In the cortical compartment, with PTH therapy there is increased cortical porosity without change in cortical width [18, 23]. This is in contrast to what is seen when PTH is given for osteoporosis, where cortical thickness increases without any change to cortical porosity [28]. In addition, there is an increase in the number of Haversian canals and the cortex becomes trabecularized. 
With PTH treatment in hypoparathyroidism, the thinning of cortical and trabecular bone may signal improved microstructural qualities since these two compartments are so abnormal prior to treatment. This is a different paradigm from PTH treatment in osteoporosis in which thickening of cortical and trabecular bone is beneficial.

\section{Safety}

If hypercalcemia occurs with PTH therapy, it tends to become evident within the first few months of treatment. It is readily corrected with titration of the patient's supplementation regimen [11-13]. Osteosarcoma has been of concern due to toxicity noted in rats given PTH at large doses and for a time period equivalent to a rat's lifespan, namely 2 years [29, 30]. This rat toxicity, however, does not appear to be a human one. There has been no increase in the incidence of osteosarcoma in patients taking PTH(1-34) over 10 years of experience [31, 32]. In the REPLACE trial, there were no significant differences in mean cardiovascular variables (blood pressure, heart rate, or QTc interval) or renal variables (serum creatinine or estimated creatinine clearance) with PTH(1-84) therapy [14]. Data in cohorts of subjects treated with PTH(1-34) through 3 years $[15,16]$ and PTH(1-84) through 4 years [12] have not raised any safety concerns, although further long-term data are necessary.

\section{Future directions}

The results described above are promising, and suggest that for many hypoparathyroid patients, PTH treatment will improve their management and outcome. Patients who can be managed well with the need for only modest amounts of calcium and vitamin D and with little fluctuation in their requirements over time, however, may not need PTH. The distinction between those who can or cannot be controlled on only modest amounts of calcium and vitamin D is not clearly related to whether or not they have residual parathyroid gland function.

There are conflicting results regarding whether the available PTH regimens have a significant effect on hypercalciuria and quality of life and further study into these areas is necessary. A major limitation of the above studies is that they have examined mostly surrogate endpoints. Further investigation is needed regarding the effects of PTH therapy on neurocognition, fractures, and more clinically relevant renal endpoints such as nephrocalcinosis and nephrolithiasis.

Other delivery systems of PTH and PTH analogs that do not require subcutaneous injection and may be more acceptable to patients are being studied in osteoporosis [33, 34] and may be applied to a hypoparathyroid population in the future. The current subcutaneous regimens do not replace PTH in a physiologic manner. More physiologic delivery systems, such as continuous delivery by pump, deserve further investigation. Further study in children with developing skeletons is necessary. Since therapy with PTH may well become a long-term management option in hypoparathyroidism, more long-term safety data are needed. 


\section{Summary}

PTH therapy in hypoparathyroidism has the potential to address aspects of the disease not ameliorated by conventional therapy. Both PTH(1-34) and PTH(1-84) have been demonstrated to lower or eliminate supplemental calcium and vitamin $D$ requirements while maintaining serum calcium. $\mathrm{PTH}(1-34)$ and $\mathrm{PTH}(1-84)$ either maintain or lower urinary calcium but more data are needed, especially using more physiologic modes of PTH replacement. PTH(1-84) has been shown to be associated with improved quality of life when the dose is titrated to serum calcium, although here as well further data are necessary. PTH(1-34) and PTH(1-84) are associated with improvement in bone-remodeling dynamics and return of bone metabolism toward more normal euparathyroid levels.

PTH may soon become an established replacement therapy for hypoparathyroidism. For the group of patients that maintain good control on calcium and active vitamin D supplementation, this development may not be needed. On the other hand, PTH may provide great benefit for patients whose management presents a challenge with conventional therapy. Discussion between the patient and care provider is necessary to determine those who may potentially benefit from PTH therapy for hypoparathyroidism, especially given the projected costs of PTH treatment.

\section{Acknowledgments}

The authors would like to acknowledge the following funding sources: National Institutes of Health grants DK069350 and DK095944 and Food and Drug Administration grant 002525.

Dr. Bilezikian is a consultant for Eli Lilly, NPS Pharmaceuticals, Merck, Amgen, and receives research support from NPS Pharmaceuticals and Amgen. Dr. Rubin receives research support from NPS Pharmaceuticals.

Role of the funding source

The study sponsors had no role in the collection, analysis and interpretation of data or in the writing of the manuscript.

\section{References}

1. Bilezikian JP, Khan A, Potts JT Jr, et al. Hypoparathyroidism in the adult: epidemiology, diagnosis, pathophysiology, target-organ involvement, treatment, and challenges for future research. Journal of Bone and Mineral Research. 2011; 26:2317-2337. [PubMed: 21812031]

2. Cusano NE, Rubin MR, Sliney J Jr, et al. Mini-review: new therapeutic options in hypoparathyroidism. Endocrine. 2012; 41:410-414. [PubMed: 22311174]

3. Shoback D. Clinical practice. Hypoparathyroidism. New England Journal of Medicine. 2008; 359:391-403. [PubMed: 18650515]

4. Mitchell DM, Regan S, Cooley MR, et al. Long-term follow-up of patients with hypoparathyroidism. Journal of Clinical Endocrinology and Metabolism. 2012; 97:4507-4514. [PubMed: 23043192]

5. Mendonca ML, Pereira FA, Nogueira-Barbosa MH, et al. Increased vertebral morphometric fracture in patients with postsurgical hypoparathyroidism despite normal bone mineral density. BMC Endocrine Disorders. 2013; 13:1. [PubMed: 23286605]

6. Underbjerg L, Sikjaer T, Mosekilde L, et al. Post-Surgical Hypoparathyroidism - Risk of Fractures, Psychiatric Diseases, Cancer, Cataract, and Infections. Journal of Bone and Mineral Research. 2014 (In press). 
7. Powers J, Joy K, Ruscio A, et al. Prevalence and incidence of hypoparathyroidism in the United States using a large claims database. Journal of Bone and Mineral Research. 2013; 28:2570-2576. [PubMed: 23737456]

8. Pearce SH, Williamson C, Kifor O, et al. A familial syndrome of hypocalcemia with hypercalciuria due to mutations in the calcium-sensing receptor. New England Journal of Medicine. 1996; 335:1115-1122. [PubMed: 8813042]

9. Sunthornthepvarakul T, Churesigaew S, Ngowngarmratana S. A novel mutation of the signal peptide of the preproparathyroid hormone gene associated with autosomal recessive familial isolated hypoparathyroidism. Journal of Clinical Endocrinology and Metabolism. 1999; 84:37923796. [PubMed: 10523031]

10. Albright F, Ellsworth R. STUDIES ON THE PHYSIOLOGY OF THE PARATHYROID GLANDS: I. Calcium and Phosphorus Studies on a Case of Idiopathic Hypoparathyroidism. Journal of Clinical Investestigation. 1929; 7:183-201.

11. Rubin MR, Sliney J Jr, McMahon DJ, et al. Therapy of hypoparathyroidism with intact parathyroid hormone. Osteoporosis International. 2010; 21:1927-1934. [PubMed: 20094706]

12. Cusano NE, Rubin MR, McMahon DJ, et al. Therapy of Hypoparathyroidism with PTH(1-84): A Prospective Four-Year Investigation of Efficacy and Safety. Journal of Clinical Endocrinology and Metabolism. 2013; 98:137-144. [PubMed: 23162103]

13. Sikjaer T, Rejnmark L, Rolighed L, et al. The effect of adding PTH(1-84) to conventional treatment of hypoparathyroidism: a randomized, placebo-controlled study. Journal of Bone and Mineral Research. 2011; 26:2358-2370. [PubMed: 21773992]

14. Mannstadt M, Clarke BL, Vokes T, et al. Efficacy and safety of recombinant human parathyroid hormone (1-84) in hypoparathyroidism (REPLACE): a double-blind, placebo-controlled, randomised, phase 3 study. The Lancet Diabetes \& Endocrinology. 2013; 1:275-283. [PubMed: 24622413]

15. Winer KK, Ko CW, Reynolds JC, et al. Long-term treatment of hypoparathyroidism: a randomized controlled study comparing parathyroid hormone-(1-34) versus calcitriol and calcium. Journal of Clinical Endocrinology and Metabolism. 2003; 88:4214-4220. [PubMed: 12970289]

16. Winer KK, Sinaii N, Reynolds J, et al. Long-term treatment of 12 children with chronic hypoparathyroidism: a randomized trial comparing synthetic human parathyroid hormone 1-34 versus calcitriol and calcium. Journal of Clinical Endocrinology and Metabolism. 2010; 95:26802688. [PubMed: 20392870]

17. Winer KK, Zhang B, Shrader JA, et al. Synthetic Human Parathyroid Hormone 1-34 Replacement Therapy: A Randomized Crossover Trial Comparing Pump Versus Injections in the Treatment of Chronic Hypoparathyroidism. Journal of Clinical Endocrinology and Metabolism. 2011; 97:391399. [PubMed: 22090268]

18. Gafni RI, Brahim JS, Andreopoulou P, et al. Daily parathyroid hormone 1-34 replacement therapy for hypoparathyroidism induces marked changes in bone turnover and structure. Journal of Bone and Mineral Research. 2012; 27:1811-1820. [PubMed: 22492501]

19. Arlt W, Fremerey C, Callies F, et al. Well-being, mood and calcium homeostasis in patients with hypoparathyroidism receiving standard treatment with calcium and vitamin D. European Journal of Endocrinology. 2002; 146:215-222. [PubMed: 11834431]

20. Cusano NE, Rubin MR, McMahon DJ, et al. The effect of PTH(1-84) on quality of life in hypoparathyroidism. Journal of Clinical Endocrinology and Metabolism. 2013; 98:2356-2361. [PubMed: 23596139]

21. Sikjaer T, Rolighed L, Hess A, et al. Effects of PTH(1-84) therapy on muscle function and quality of life in hypoparathyroidism: results from a randomized controlled trial. Osteoporosis International. 2014; 25:1717-1726. [PubMed: 24687385]

22. Cusano NE, Rubin MR, McMahon DJ, et al. PTH(1-84) is associated with improved quality of life in hypoparathyroidism Through 5 Years of Therapy. Journal of Clinical Endocrinology and Metabolism. 2014 (In press).

23. Rubin MR, Dempster DW, Sliney J Jr, et al. PTH(1-84) administration reverses abnormal boneremodeling dynamics and structure in hypoparathyroidism. Journal of Bone and Mineral Research. 2011; 26:2727-2736. [PubMed: 21735476] 
24. Rubin MR, Dempster DW, Zhou H, et al. Dynamic and structural properties of the skeleton in hypoparathyroidism. Journal of Bone and Mineral Research. 2008; 23:2018-2024. [PubMed: 18684087]

25. Sikjaer T, Rejnmark L, Thomsen JS, et al. Changes in 3-dimensional bone structure indices in hypoparathyroid patients treated with $\mathrm{PTH}(1-84)$ : a randomized controlled study. Journal of Bone and Mineral Research. 2012; 27:781-788. [PubMed: 22161686]

26. Recker RR, Bare SP, Smith SY, et al. Cancellous and cortical bone architecture and turnover at the iliac crest of postmenopausal osteoporotic women treated with parathyroid hormone 1-84. Bone. 2009; 44:113-119. [PubMed: 18983947]

27. Jobke B, Muche B, Burghardt AJ, et al. Teriparatide in bisphosphonate-resistant osteoporosis: microarchitectural changes and clinical results after 6 and 18 months. Calcified Tissue International. 2011; 89:130-139. [PubMed: 21626160]

28. Dempster DW, Cosman F, Kurland ES, et al. Effects of daily treatment with parathyroid hormone on bone microarchitecture and turnover in patients with osteoporosis: a paired biopsy study. Journal of Bone and Mineral Research. 2001; 16:1846-1853. [PubMed: 11585349]

29. Vahle JL, Sato M, Long GG, et al. Skeletal changes in rats given daily subcutaneous injections of recombinant human parathyroid hormone (1-34) for 2 years and relevance to human safety. Toxicologic Pathology. 2002; 30:312-321. [PubMed: 12051548]

30. Jolette J, Wilker CE, Smith SY, et al. Defining a noncarcinogenic dose of recombinant human parathyroid hormone 1-84 in a 2-year study in Fischer 344 rats. Toxicologic Pathology. 2006; 34:929-940. [PubMed: 17178693]

31. Andrews EB, Gilsenan AW, Midkiff K, et al. The US postmarketing surveillance study of adult osteosarcoma and teriparatide: Study design and findings from the first 7 years. Journal of Bone and Mineral Research. 2012; 27:2429-2437. [PubMed: 22991313]

32. Cipriani C, Irani D, Bilezikian JP. Safety of osteoanabolic therapy: a decade of experience. Journal of Bone and Mineral Research. 2012; 27:2419-2428. [PubMed: 23165426]

33. Daddona PE, Matriano JA, Mandema J, et al. Parathyroid hormone (1-34)-coated microneedle patch system: clinical pharmacokinetics and pharmacodynamics for treatment of osteoporosis. Pharmaceutical Research. 2011; 28:159-165. [PubMed: 20567999]

34. Hattersley, G.; Bilezikian, JP.; Guerriero, J., et al. Bone Anabolic Efficacy and Safety of BA058, a Novel Analog of hPTHrP: Results from a Phase 2 Clinical Trial in Postmenopausal Women with Osteoporosis. The Endocrine Society's 94th Annual Meeting and Expo; Houston, TX. OR08-12012

35. Winer KK, Yanovski JA, Sarani B, et al. A randomized, cross-over trial of once-daily versus twice-daily parathyroid hormone 1-34 in treatment of hypoparathyroidism. Journal of Clinical Endocrinology and Metabolism. 1998; 83:3480-3486. [PubMed: 9768650]

36. Winer KK, Sinaii N, Peterson D, et al. Effects of once versus twice-daily parathyroid hormone 134 therapy in children with hypoparathyroidism. Journal of Clinical Endocrinology and Metabolism. 2008; 93:3389-3395. [PubMed: 18492754] 


\section{Practice Points}

- Conventional therapy of hypoparathyroidism includes calcium and active vitamin D supplementation, often in large doses

- The potential advantages of PTH over conventional therapy in the management of hypoparathyroidism include: a reduction in the amounts of calcium and vitamin D requirements, reduction in urinary calcium, improvement in quality of life and improvement in abnormal bone remodeling dynamics

- PTH may soon become an established replacement therapy for hypoparathyroidism

- PTH therapy may not be suitable for all patients, either due to clinical circumstances or due to cost

\section{Research Agenda}

- Other delivery systems of PTH and PTH analogs that do not require subcutaneous injection are under investigation in osteoporotic subjects and may be applied to a hypoparathyroid population in the future

- More physiologic delivery systems, such as continuous delivery by pump, deserve further investigation

- Further study in children with developing skeletons is necessary

- Since therapy with PTH may well become a long-term management option in hypoparathyroidism, more long-term safety data are needed 


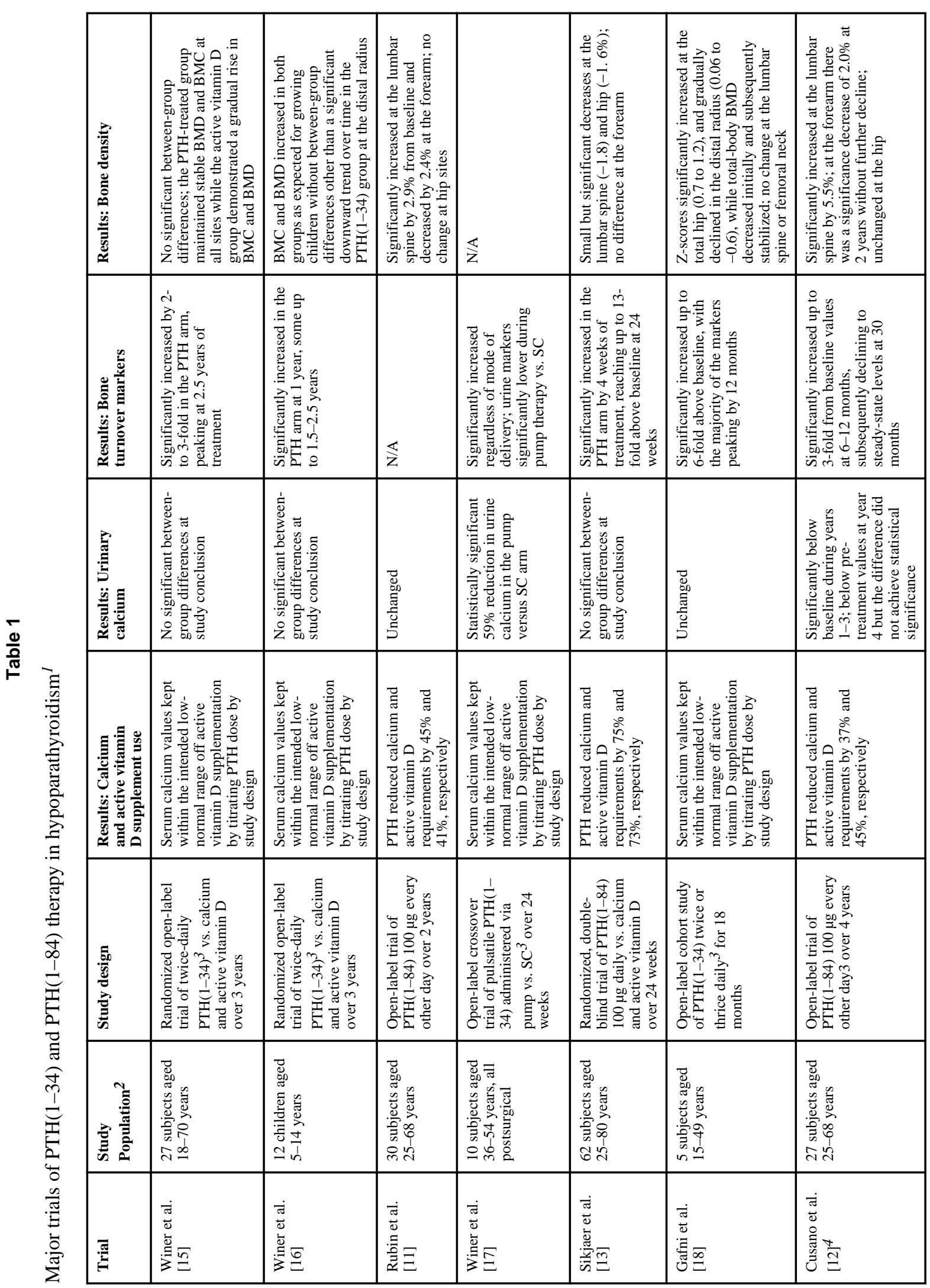




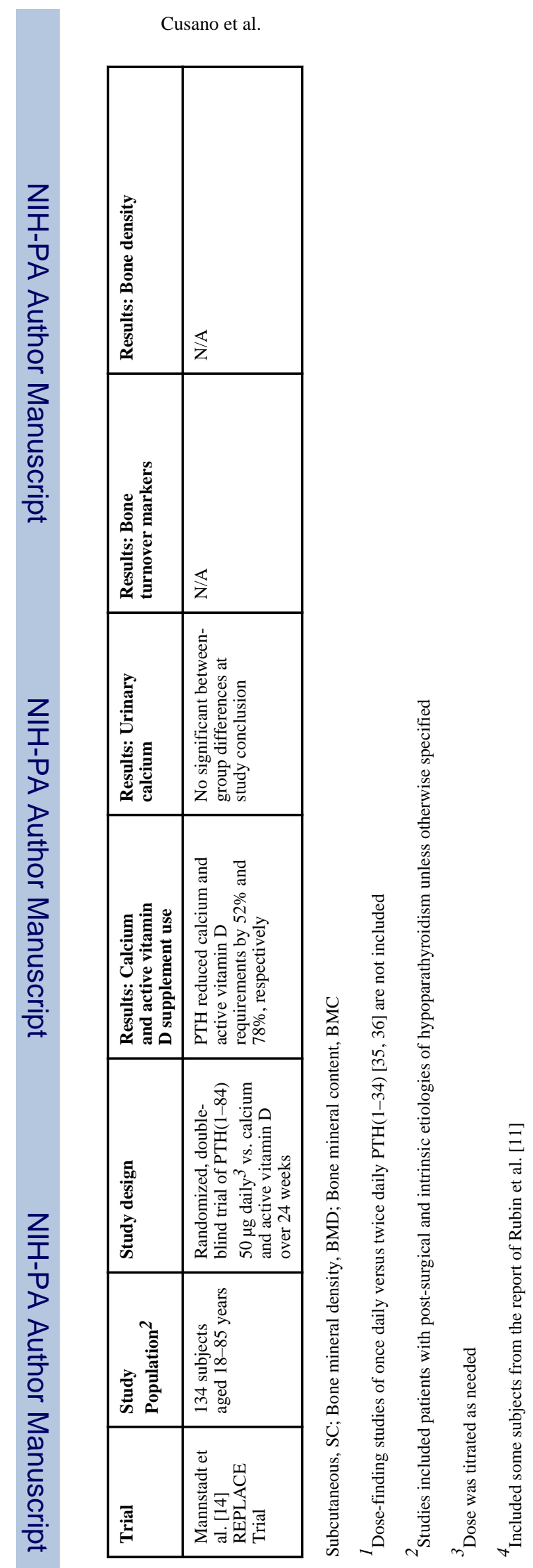

Page 12 